\title{
Systematic Studies on Hill Stream Cyprinid Fishes of Manimala River of Kerala, India
}

\author{
*Mathews Plamoottil \\ Asst. Professor \& HOD, Dept. of Zoology, \\ Baby John Memorial Govt. College, Chavara, Kollam, Kerala \\ Ashim Kumar Nath \\ Professor, Department of Zoology, Sidho Kanho Birsha University \\ Purulia, West Bengal \\ *mathewsplamoottil@gmail.com
}

\begin{abstract}
The present study was conducted to analyze the taxonomic details of cyprinid fishes occurring in the high level regions of Manimala River of Kerala, India. Different species of cyprinid fishes were collected from different sites of the river. 12 species of fishes belong to the 11 genera and three families were collected from various locations. Systematic studies were conducted on these fishes.
\end{abstract}

Keywords: Travancore, Freshwater fishes, Taxonomy, Distribution, Puntius

Abbreviations: D-Dorsal fin rays; A-Anal fin rays; $V$ - Ventral fin rays; $P$ - pectoral fin rays; $C$ - Caudal fin rays; LLS-Lateral line scales; PRD-Pre dorsal scales; PRPL-Pre pelvic scales; PRA-pre anal scales; HDHead depth; HW- Head width; BDD-Body depth at dorsal fin origin; BWD-Body width at dorsal fin; BWVBody width at ventral fin; PRD-Pre dorsal distance; PRP-Pre pectoral distance; PRA-Pre anal distance; $P R P L$ - Pre pelvic distance; LP- length of pectoral fin; $L P L$ - Length of pelvic fin; LA-length of anal fin; LClength of caudal fin; $D V V$-Distance from ventral to vent; $B D V$-Body depth at ventral fin; DP-PL-Distance from pectoral to pelvic; DPL-A-Distance from pelvic to anal fin; DA-C-Distance from anal to caudal; ED-Eye diameter; IOW-Inter orbital width; STL-Snout length; WGM-Width of gape of mouth; INW-Inter narial width.

\section{INTRODUCTION}

Manimala River in Kerala, India is a small west flowing mountainous river, originating from Thattemalai hills of the Western Ghats mountain ranges at an elevation of $1156 \mathrm{~m}$ above MSL. It is with a length of about $90 \mathrm{~km}$ and a catchment area of about $847 \mathrm{Km}^{2}$. It is short and torrential due to heavy rainfall and steep gradient. The complex topography, high rainfall, wide altitudinal variation and warm humid tropical climate have produced a variety of ecological niches with unique instream and riparian plants and animals.

At High level regions, Manimala River flows through Idukki and Kottayam districts of Kerala. Kokkayar, Pullaga (Pullaraaar) and Kanjirappallythodu (also known as Chittaar) are the main tributaries of this river in high land regions. At high lands Manimala River flows through ElankaduTop, Elankad, Yendayar, Thenpuzha, Kuppayakkuzhy, Koottickal, Velanilam, Nenmeni, Mundakkayam, Vallakkadavu and Koratti (Erumeli). River basin is characterized by denudational hills, piedmont zones, residual mounts and valley hills. It consists of forest lands, scrub lands and agricultural lands. High altitude areas are used mainly for rubber plantations. High level regions of Manimala River are characterized by intermittent occurrence of moderately dense riparian vegetation.

Manimala River is rich in ichthyofaunal resources. Plamoottil [1] reported ninety seven species of fishes in this river. Many new fishes had also been described from high level regions of Manimala River. Day [2] drescribed and named Mesonoemocheilus triangularis, Barilius bakeri and Puntius denisoni (=Sahyadria denisoni) from Mundakkayam of Manimala River. His Hara malabarica (= Mystus canarensis) was also from 'Hill Ranges of Travancore'. Gunther [3] described Catopra malabarica (=Pristolepis malabarica) from hilly areas of Manimala River. Plamoottil and Abraham $[4,5,6]$ discovered, named and described Glyptothorax elankadensis, Mystus menoni and M. heoki from high level regions of the river. From the literature it was understood that no serious taxonomic 
studies were conducted on the hill stream cyprinid fishes of Manimala River; so it is an endeavor to study the systematic aspects of cyprinid fishes of Manimala River.

\section{Materials AND Methods}

Different species of cyprinid fishes were collected from various selected sites of high level regions using gill net, cast net, madavala, vattavala etc. Selection of these gears is based on its fishing efficiency in a running water body. Collected fishes were kept in $10 \%$ formalin, identified and taxonomically analyzed.

\section{RESUltS}

Fishes belonging to the families Danionidae, Cyprinidae and Balitoridae were collected from high level regions (Fig. 1- 7) of Manimala River. They were found to be species of the genera Salmostoma, Barilius, Devario, Dawkinsia, Rasbora, Puntius, Gonoproktopterus, Garra, Haludaria, Mesonoemacheilus and Bhavania (Fig. 8- 19; Table $1 \&$ 2). Of these, Dawkinsia was the edible cyprinid fish abundantly distributed in all high level regions of the river. Barilius bakeri and Dawkinsia filamentosa were also collected from all stations.

\subsection{Salmostoma boopis (Day)}

Chela boopis Day,1873,Proc. Zool. Soc. London, 708 (type locality: South Canara).

Diagnosis: Anal fin with thirteen branched rays; lateral line curved and complete with 39 scales. Dorsal fin inserted in advance of anal fin; no barbels.

Meristic counts:D- 7; A- 11; V- i, 5; P- 11; C- 17; LLS- 40; LL/V- 11/2; LL/A-1/2; LL/D- 51/2; PRD- 25; PRPL- 11; PRA- 20.

Metric characters:TL (mm)- 123.0; SL (mm)- 98.0; HL (mm) - 23.0. \% SL: HL-23.5; HD- 16.3; HW12.2; BDD-17.3; BWD-9.2; BWV-11.2; PRD-66.3; PRP-24.5; PRA- 69.4; PRPL- 50.0; LP- 25.5; LPL-14.3; LA- 12.2; LC- 26.0; DVV-18.4; BDV-20.4; DP-PL-26.5; DPL-A-21.4; DA-C- 28.6. \% HL: HD- 69.6; HW-52.2; ED-39.1; IOW-30.4; STL-30.4; WGM-21.7; INW-21.7.

Other features: Lower jaw upwardly directed with well developed symphyseal process. Gape of mouth reach to little behind the front border of the orbit; eyes large; pectorals elongated and it reach pelvic fin; dorsal and anal fins placed at the posterior part of the body; an indistinct mid lateral bluish black line present from middle part to posterior tip; minute black spots present all over the body.

Remarks: It is abundant in high level and middle level regions of Manimala River. It showed some meristic and metric differences from Salmostoma boopis collected from Wayanad. Further research works alone can help to reach to a conclusion.

\subsection{Barilius bakeri Day}

Barilius bakeri Day, 1865, Proc. Zool. Soc. London, 305 (type locality; Mundakkayam, Kerala).

Diagnosis: Lower jaw with a knob at its extremity. Single row of 9-11 large metallic silvery bluish black spots along sides; deep body; barbels absent; maxilla reaches below orbit.

Meristic counts: D- ii, 10; P- i, 14; V- i, 8; A- iii, 14; C- 17; LLS- 37; PDS- 14-16; PRPLS- 15; PRAS- 23; LL/D- 10; LL/V- 31/2; L/TR- 10/31/2; LL/A- 31/2; CPS- 7.

Metric characters: TL (mm)- 135.0; SL (mm)-110.0; HL (mm) - 30.0.

\% SL: HL- 27.3; HD- 21.8; HW- 15.0; BDD- 34.5; BDA- 24.7; BWD-17.3; BWA-14.5; PRD-54.5; POD- 50.0; PRP-27.3; PRPL-49.0; PRA- 64.5; LD- 16.4; LP- 18.6; LPL-12.5; LA- 15.5; LBD- 16.8; LBA- 20.0; LBP-5.0; LBPL-4.5; LCP- 12.7; DCP- 10.5; WCP- 6.4; DAV-16.4; DVV- 16.4. \% HL: HD-80.0; HW- 55.0; ED- 23.3; IOW-33.3; INW- 23.3; WGM- 26.7; STL-30.0

Other features: Gape of mouth large. Lateral line strongly concave. Scales larger. Dorsal fin without scales at its base; but that of anal scaled. Caudal deeply forked; the lower lobe longer. Dorsal side of the body bluish; ventral side white; bases of dorsal, anal and pectoral fins dark; their margins white.

Remarks: In the specimens collected from Erumely 9 black lateral spots present on one side and 11 spots on the other side. Elankadu and Mundakkayam specimens are with 10 spots on either side. These mid lateral spots spherical in the fishes collected from Erumely and Elankadu; but in those of Mundakkayam and Chenappady they are spindle shaped or roughly elongated. 


\subsection{Devario(Danio) aequipinnatus (McClelland)}

Perilampus aequipinnatus McClelland, 1839, Asiat. Res.., 19 (2): 393 (type locality: Assam).

Diagnosis: 14- 15 scales present before the dorsal fin; lateral line scales 35- 37; three well marked dark blue bands run along the flanks through the entire length from head to caudal fin.

Meristic counts: D- iii, 9- 12; P- I, 11- 12; V- I, 6; A- iii, 14- 16; LLS- 35- 37.

Metric characters: TL (mm) - 95.0 SL (mm) - 74.3; HL (mm)-10.6. \% SL: HL-21.5; HD-18.8; HW10.8; BDD-29.6; BDA-21.5; BWD-10.8; BWA-8.1; PRD-56.5; PRA-71.3; PRPL-47.1; PL-22.8; LPL-15.5; LA-14.8; LC-30.9; LBD-20.2; LBA-21.5; LCP-17.8; DCP-10.8; DP-PL- 29.6; DPL- A22.8; DA- C-36.3;\% HL: HD- 87.5; HW-10.8; STL-32.5; WGM-25.0; IOW-27.0; INW-23.0.

Other features: Ground color blue to light brown; a well defined blotch present at the upper angle of the gill opening.

\subsection{Devario(Danio) malabaricus (Jerdon)}

Perilampus malabaricus Jerdon, 1849, Madras J. Lit.and Sci., 15: 325. (Type locality: Malabar).

Diagnosis: Lateral line complete with 32- 34 scales; fourteen to fifteen scales are present before the dorsal fin; three well- marked lateral bands of dark blue color run along the sides covering along the entire length from head to caudal fin.

Meristic counts: D- iii, 13; P- i, 14; V- i, 7; A- iii, 13; C 16; LLS- 33; PDS 16; PRPLS 8; PRAS 15.

Metric characters: TL (mm) - 95.5; SL (mm) - 74.0; HL (mm) - 17.0. \% SL: HL-22.9; HD-17.6; HW12.2; BDD-28.4; BDA-24.3; BWD-10.8; BWA-8.4; PRD- 58.1; PRA-68.9; PRPL-47.3; LP-23.0; LPL-15.5; LA-16.2; LC-29.1; LBD-19.6; LBA-21.6; LCP-17.8; DCP-10.8; DP-PL-27.7; DPL- A22.9; DA- C-37.8. \% HL: ED-35.3; STL-32.3; WGM-26.5; IOW-28.2; INW-23.5.

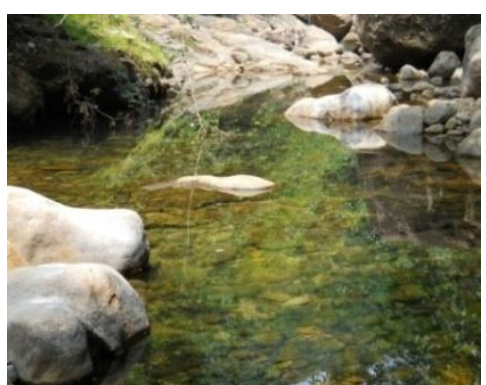

Fig. 1 Elankadu

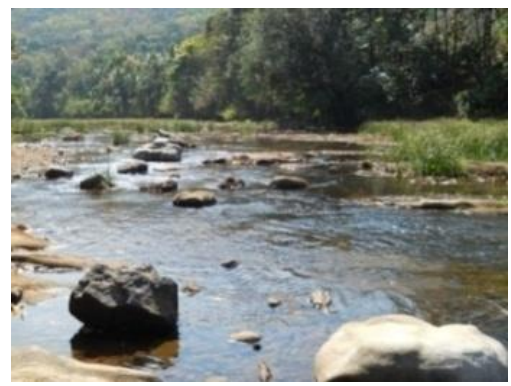

Fig. 4 Velanilam

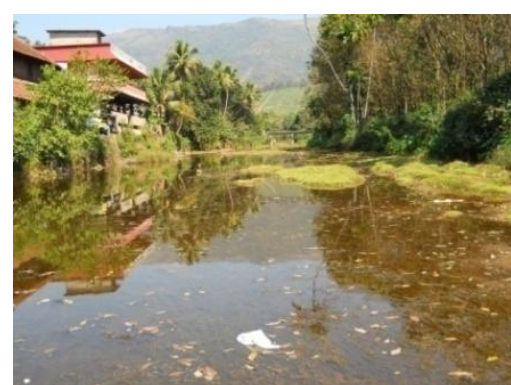

Fig. 2 Yendayar

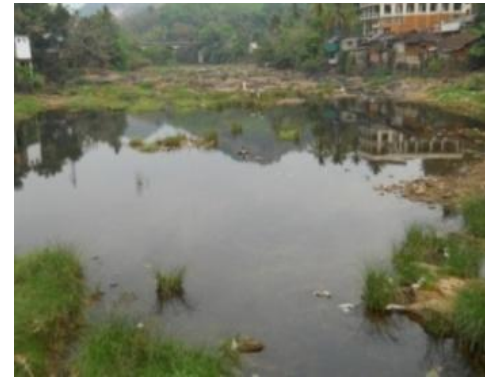

Fig.5 Mundakkayam

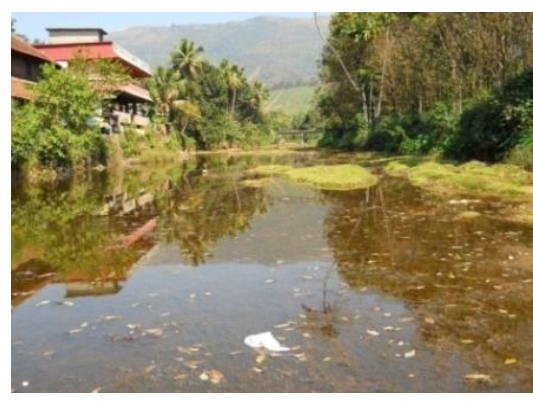

Fig. 3 Koottickal

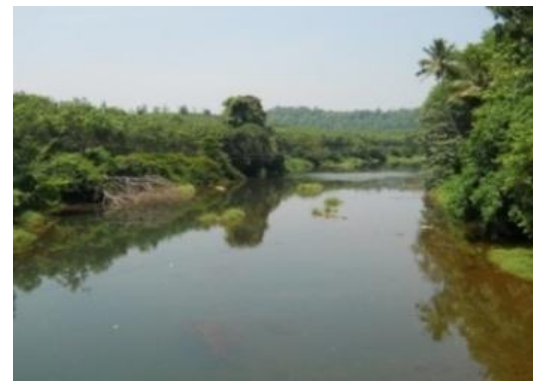

Fig.6 Vallakkadavu

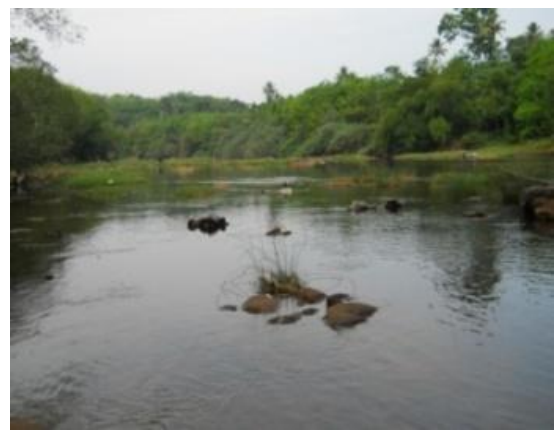

Fig. 7 Orumkal 


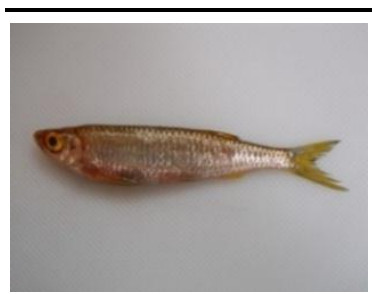

Fig. 8 S. boopis

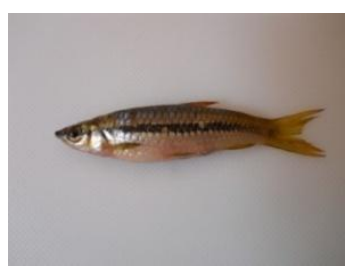

Fig. 12 R. daniconius

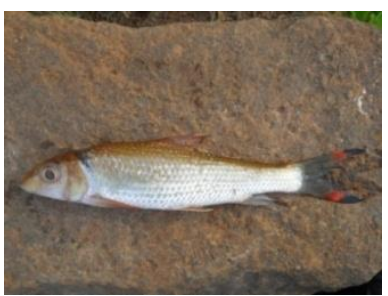

Fig. 16 G. kurali

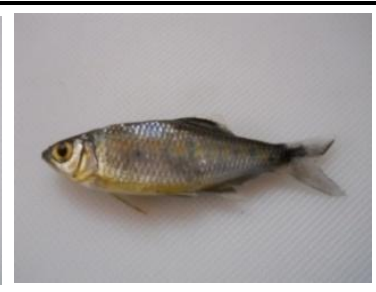

Fig. 9 B. bakeri

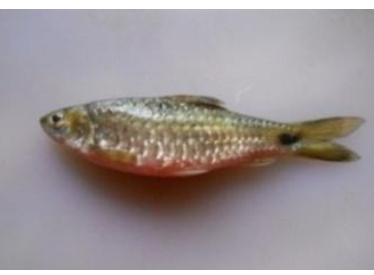

Fig. 13 P. mahecola

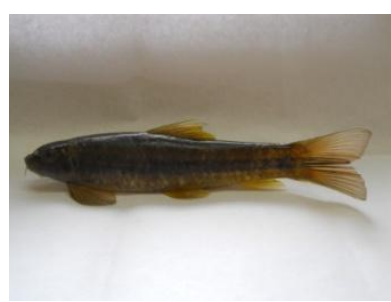

Fig. 17 Garra mully

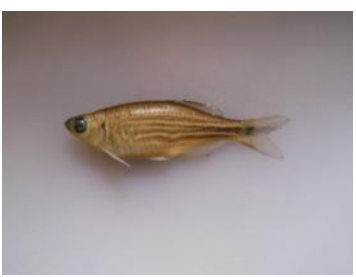

Fig. 10 D. aequipinnatus

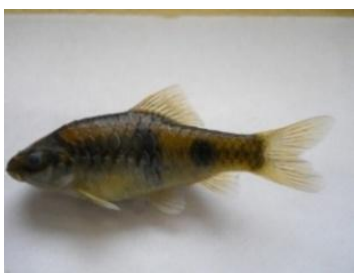

Fig. $14 H$. fasciatus

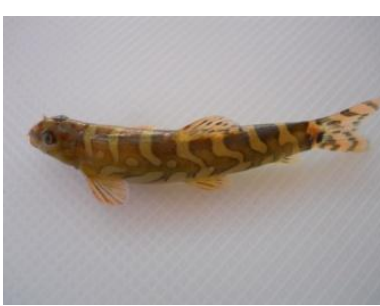

Fig. 18 M. triangularis

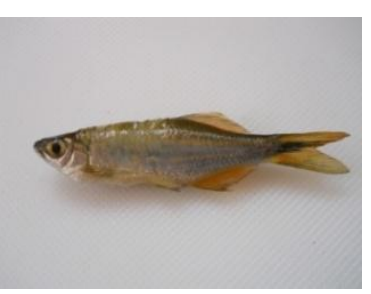

Fig. 11 D. malabaricus

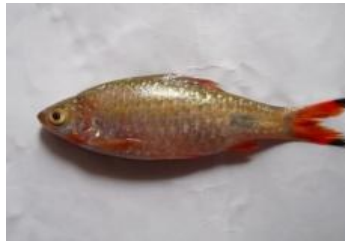

Fig. 15 D. filamentosa

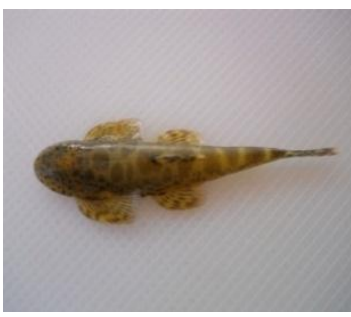

Fig. 19 B. australis

Table1. Distribution of cyprinid fishes in high level regions of Manimala River

\begin{tabular}{|l|l|c|c|c|c|c|c|c|}
\hline Sl. no & Fishes & Elankadu & Yendayar & Koottikal & Velanilam & Mundakkaym & Vallakkadau & Orumkal \\
\hline 1 & $\begin{array}{l}\text { Salmophasia } \\
\text { boopis }\end{array}$ & & & & & + & + & + \\
\hline 2 & Barilius bakeri & + & + & + & + & + & + & + \\
\hline 3 & $\begin{array}{l}\text { Devario } \\
\text { aequipinnatus }\end{array}$ & & & & & + & + & + \\
\hline 4 & $\begin{array}{l}\text { Devario } \\
\text { malabaricus }\end{array}$ & + & + & & + & + & + & + \\
\hline 5 & $\begin{array}{l}\text { Rasbora } \\
\text { daniconius }\end{array}$ & + & + & + & + & + & + \\
\hline 6 & $\begin{array}{l}\text { Puntius } \\
\text { mahecola }\end{array}$ & $\begin{array}{l}\text { Haludaria } \\
\text { fasciatus }\end{array}$ & + & + & + & + & + & + \\
\hline 8 & $\begin{array}{l}\text { Dawkinsia } \\
\text { filamentosa }\end{array}$ & + & + & + & + & + & + \\
\hline 9 & $\begin{array}{l}\text { Gonoproktopteru } \\
\text { surali }\end{array}$ & & + & & & + & + & + \\
\hline 10 & Garra mullya & + & + & + & + & + & + & + \\
\hline 11 & $\begin{array}{l}\text { Mesonoemacheil } \\
\text { us traiangularis }\end{array}$ & + & + & & & + & + & + \\
\hline 12 & $\begin{array}{l}\text { Bhavania } \\
\text { australis }\end{array}$ & & & & & + & + \\
\hline
\end{tabular}

Table2. Classification, names and systematic position of hill stream cyprinid fishes of Manimala River

\begin{tabular}{|c|c|c|c|c|c|c|c|}
\hline Sl.no. & Name & Order /Family & Synonyms & $\begin{array}{l}\text { Common } \\
\text { names }\end{array}$ & $\begin{array}{l}\text { Regional } \\
\text { names }\end{array}$ & $\begin{array}{l}\text { Economic } \\
\text { value }\end{array}$ & $\begin{array}{l}\text { IUCN } \\
\text { Status }\end{array}$ \\
\hline 1 & $\begin{array}{l}\text { Salmostoma } \\
\text { boopis }\end{array}$ & $\begin{array}{l}\text { Cypriniformes } \\
\text { Danionidae }\end{array}$ & Chela boopis & $\begin{array}{l}\text { Boopis razor } \\
\text { belly- minnow }\end{array}$ & $\begin{array}{l}\text { Mathiparal, } \\
\text { Kathiparal, } \\
\text { Wayanadan- } \\
\text { mathi, } \\
\text { Alari }\end{array}$ & $\mathrm{FF} / \mathrm{OF}$ & LRlc \\
\hline 2 & Barilius bakeri & $\begin{array}{l}\text { Cypriniformes } \\
\text { Danionidae }\end{array}$ & Nil & $\begin{array}{l}\text { Malabar baril, } \\
\text { Blue dotted hill } \\
\text { trout }\end{array}$ & $\begin{array}{l}\text { Paraloli, } \\
\text { Velingil, } \\
\text { Poovalchi, } \\
\text { Pavukan }\end{array}$ & $\mathrm{OF}$ & LRnt \\
\hline & Devario & Cypriniformes & Perilampus & Giant danio, & ThuppalKothi & $\mathrm{FF}, \mathrm{OF}$ & LRlc \\
\hline
\end{tabular}


Systematic Studies on Hill Stream Cyprinid Fishes of Manimala River of Kerala, India

\begin{tabular}{|c|c|c|c|c|c|c|c|}
\hline 3 & aequipinnatus & Danionidae & \begin{tabular}{|l} 
aequipinnatus \\
Danio browni \\
Danio strigillifer \\
Danio deyi
\end{tabular} & $\begin{array}{l}\text { Blue danio, } \\
\text { Giant danio }\end{array}$ & & & \\
\hline 4 & $\begin{array}{l}\text { Devario } \\
\text { malabaricus }\end{array}$ & $\begin{array}{l}\text { Cypriniformes } \\
\text { Danionidae }\end{array}$ & $\begin{array}{l}\text { Perilampus } \\
\text { malabaricus, } \\
\text { Eustira ceylonensis, } \\
\text { Perilampus } \\
\text { ceylonensis, }\end{array}$ & Malabar danio & ThuppalKothi & $\mathrm{FF}, \mathrm{OF}$ & LRlc \\
\hline 5 & $\begin{array}{l}\text { Rasbora } \\
\text { daniconius }\end{array}$ & $\begin{array}{l}\text { Cypriniformes } \\
\text { Danionidae }\end{array}$ & $\begin{array}{l}\text { Cyprinus daniconius, } \\
\text { Parluciosoma } \\
\text { daniconius }\end{array}$ & \begin{tabular}{|lr} 
Black line \\
rasbora, \\
Slender & \\
& \\
\end{tabular} & $\begin{array}{l}\text { Thuppal-Kothi, } \\
\text { Kodiyanparal, } \\
\text { Kanangon }\end{array}$ & $\mathrm{OF}$ & LRnt \\
\hline 6 & $\begin{array}{l}\text { Puntius } \\
\text { mahecola }\end{array}$ & $\begin{array}{l}\text { Cypriniformes } \\
\text { Cyprinidae }\end{array}$ & $\begin{array}{l}\text { Leuciscus mahecola } \\
\text { Barbus mahecola }\end{array}$ & Malini barb & Urulanparal & FF, OF & $\mathrm{E}$ \\
\hline 7 & $\begin{array}{l}\text { Haludaria } \\
\text { fasciatus }\end{array}$ & $\begin{array}{l}\text { Cypriniformes } \\
\text { Cyprinidae }\end{array}$ & $\begin{array}{l}\text { Cirrhinus fasciatus } \\
\text { Puntius fasciatus } \\
\text { P.f. pradhani }\end{array}$ & Melon barb & $\begin{array}{l}\text { Vazhakkava- } \\
\text { rayan, } \\
\text { Vazhakkaparal, } \\
\text { Cheruparal }\end{array}$ & $\mathrm{OF}$ & LRnt \\
\hline 8 & $\begin{array}{l}\text { Dawkinsia } \\
\text { filamentosa }\end{array}$ & $\begin{array}{l}\text { Cypriniformes } \\
\text { Cyprinidae }\end{array}$ & \begin{tabular}{|l|} 
Leuciscus \\
filamentosus \\
Barbus filamentosus \\
Puntius filamentosus
\end{tabular} & $\begin{array}{l}\text { Black spot barb, } \\
\text { Indian tiger barb }\end{array}$ & $\begin{array}{l}\text { Poovaliparal, } \\
\text { Pullipparal, } \\
\text { Valelchutty- } \\
\text { paral, } \\
\text { Chemparal, } \\
\text { Kalakodiyan }\end{array}$ & FF, OF & LRlc \\
\hline 9 & $\begin{array}{l}\text { Gonoproktopter } \\
\text { us kurali }\end{array}$ & $\begin{array}{l}\text { Cypriniformes } \\
\text { Cyprinidae }\end{array}$ & Hypselobarbus kurali & Nil & Kooral & FF, OF & EN \\
\hline 10 & Garra mullya & $\begin{array}{l}\text { Cypriniformes } \\
\text { Cyprinidae }\end{array}$ & \begin{tabular}{|l} 
Chondrostoma \\
mullya \\
Discognathus lamta \\
Garra jerdonia \\
Garra \\
jenkinsonianum \\
Horalabiosajoshuai \\
Garra menoni
\end{tabular} & $\begin{array}{l}\text { Mullyagarra } \\
\text { Brown alga eater }\end{array}$ & $\begin{array}{l}\text { Kallunakki, } \\
\text { Kallemutti } \\
\text { Kallemkari } \\
\text { Kallotti, } \\
\text { Kallunthi, } \\
\text { Kodali } \\
\text { Paraenakki }\end{array}$ & $\mathrm{FF}$ & LRlc \\
\hline 11 & \begin{tabular}{|l|} 
Mesonoemache \\
ilus \\
triangularis
\end{tabular} & $\begin{array}{c}\text { Cypriniformes } \\
\text { Balitoridae, }\end{array}$ & $\begin{array}{l}\text { Nemacheilus } \\
\text { triangularis } \\
\text { Nemachilus } \\
\text { riangularis } \\
\text { N.t.triangularis } \\
\text { N.t. } \\
\text { tambaraparniensis }\end{array}$ & Zodiac loach & $\begin{array}{l}\text { Koima, } \\
\text { Koitha, } \\
\text { Aira, } \\
\text { Manalayira, } \\
\text { Manalaari }\end{array}$ & $\mathrm{OF}$ & LRnt \\
\hline 12 & $\begin{array}{l}\text { Bhavania } \\
\text { australis }\end{array}$ & $\begin{array}{l}\text { Cypriniforme } \\
\text { Balitoridae }\end{array}$ & $\begin{array}{l}\text { Platycara australis } \\
\text { Homaloptera } \\
\text { maculate } \\
\text { H. brucei } \\
\text { Bhavania annandalei }\end{array}$ & $\begin{array}{l}\text { Western Ghat } \\
\text { loach }\end{array}$ & Kalnakki & $\mathrm{OF}$ & LRnt \\
\hline
\end{tabular}

\subsection{Rasbora (Parluciosoma) daniconius (Hamilton- Buchanan)}

Cyprinus daniconius Hamilton- Buchanan, 1822, Fishes of Ganges: 327, 391 (type locality: Rivers of Southern Bengal).

Diagnosis: Barbels absent; mouth terminal and upturned; a gold bordered dark lateral band runs from eye to the base of caudal fin; lateral line complete with 32 scales, situated on the lower

lateral side; $1 \frac{1}{2}$ scales between lateral line and ventral fin; dorsal fin located nearer to caudal fin than snout tip.

Meristic counts: D- 8; P- 12; V- 9; A- 5; C-17; LLS- 32; PDS- 14; LL/D- 51/2; LL/V- 11/2; LL/A- 21/2; PRPLS- 7; PRAS- 17. 
Metric characters: TL (mm)- 93.5; SL (mm)-73.0; HL (mm)- 19.0.\% SL: HL-26.0; HD- 16.4; HW13.7; BDD-21.9; BDA-17.8; BWD-15.1; BWA-10.9; PRD-53.4; POD-45.2; PRP-26.0; PRA- 79.5; PRPL-47.9; LP- 17.8; LPL-16.4; LA-17.8; LC-28.8; DVV-23.3; DAV-2.7; DP-PL-23.3; DPL-A-26.0; DA-C-23.3; \% HL: HD- 63.2; HW-52.6; ED-31.6; WGM-26.3; STL-26.3; IOW- 31.6; INW-26.3.

\subsection{Puntius mahecola (Valenciennes)}

Leuciscus mahecolaValenciennes, 1844.Hist. Nat. Poiss.., 17: 305 (type locality, Mahe, India); Puntius mahecola, Pethiyagoda \& Kottelat, 2005, Raffles Bulletin of Zoology, 12: 145-152.

Diagnosis: Puntius mahecola is distinguished from all other Puntius species by the following combination of characters: last simple dorsal ray smooth; body depth $27.2-32.0 \%$ SL; a single pair of (maxillary) barbels, about $1 / 2$ eye diameter long; $22-23+1-3$ lateral-line scales; $1 / 24+1+31 / 2$ transverse rows of scales; a horizontally elongate black blotch about $1 \frac{1 / 2}{2}$ times as wide as it is high across $3 \frac{1}{2}$ scales of lateral line immediately behind anal-fin base.

Meristic counts: D- iii, 8; P- I, 14; V-i, 8; A- iv, 51/2; LLS- 22-23; PDS-7; LL/V-21/2; L/Tr- 51/2/31/2; CPS $-1 / 251 / 2$.

Metric characters: TL (mm)- 107.0; SL (mm)-83.0; HL (mm)- 21.0.

\% SL: HL-25.3; HD-20.5; HW-15.7; BDD-30.1; BDA-19.3; BWD-21.7; BWA-13.3; PRD-47.0; POD-56.7; PRP-26.5; PRPL-53.0; PRA-77.1; LD-21.7; LP-19.3; LPL-18.1; LA-14.5; LBD-16.8; LBA-8.4; LBP-4.8; LBPL-6.0; LCP-15.7; DCP-14.5; WCP-6.0; DAV-4.6; DVV-21.7; DP-PL-28.9; DPL-A-26.5. \% HL: HD-81.0; HW-62.0; ED-33.3; IOW-39.0; INW-23.8; WGM-33.3; STL-33.3.

Other features: Leuciscus (Puntius) mahecolaValenciennes has long been placed in the synonymy of LeuciscusfilamentosusValenciennes. Rohan Pethiyagoda and Maurice Kottelat (2005a \& 2005b) after examination of the syntype series of Puntius mahecola together with other specimens collected from Kerala showed that it is a valid species that has been referred in the current literature to Puntius amphibious (Valenciennes).

\subsection{Haludaria fasciatus (Day)}

Labeo melanampyx Day, 1865, Proc. Zool. Soc. Lond., 317; Haludaria fasciatus Pethiyagoda et al. 2012.Ichthyol.Expl. Freshwaters, 23 (1): 69-95.

Diagnosis: Last unbranched dorsal ray non osseous, weak and smooth. Lateral line with 20 scales; seven scales present before dorsal fin; three vertical bands descending up to a little below the lateral line.

Meristic counts: D- i, 8; P- I, 14; V- i,7; A- ii,5; C-16; LLS- 21; PPLS- 5; PRAS- 11; LL/D- 31/2; LL/V- 21/2; LL/A- 31/2; PDS- 7.

Metric characters: TL (mm) - 54.0; SL (mm) - 41.0; HL (mm)- 12.0.\% SL: HL-29.3; HD-24.4; HW17.1; PRD-53.6; POD-53.7; LP-22.0; LPL-22.0; LA- 19.5; LC-31.7; LBD-22.0; LBA-12.2; DP-PL26.8; DA-C-22.0; BDD-36.6; BDA-22.0; BWD-22.0; BWA-14.6; LCP-17.1; DCP-14.6; WCP-7.3. \% HL: HD-83.3; HW-58.3; ED- 33.3; WGM-33.3; STL-33.3; IOW-33.3; INW-25.0; LMB- 33.3; LRB58.3 .

Remarks: Two varieties of Haludaria fasciatus have received from Manimala River; those residing in the middle and low level regions are the common varieties. But those received from Elankadu and other high level regions exhibits marked differences; in them middle vertical band on the body forms a circle around the body and goes behind the pectoral and joins with the band of the other side. But in the first variety vertical band descending only up to a little below the lateral line. In the high land variety $\mathrm{BDD} / \mathrm{SL}$ is 3.2 , but it is 2.8 in the common variety; LL/A is $3 \frac{1}{2}$ in common variety but it is $2 \frac{1}{2}$ in high level inhabitant. More taxonomic works are required to prove that both are of different species.

\subsection{Dawkinsia filamentosa (Valenciennes)}

Leuciscu sfilamentosus Valenciennes, 1844. Hist. Nat. Poiss., 17: 495 (type locality, Alleppey, Kerala). Dawkinsia filementosa, Pethiyagoda\&Kottelat, 2005, The Raffles Bull.Zool. 12: 127- 144.

Diagnosis: Barbels are minute maxillary pair only; lateral line complete with 21 scales; a deep oval blotch present above anal fin; caudal fin with two oblique red bands bordered with black bands. 
Meristic counts: D- iii, 8; P- I, 14; V- I, 8; A- ii- iii, 5; LLS- 21; PDS-71/2; PRPLS- 9; PRAS-16; LL/D-51/2; LL/V-21/2; L/Tr- 51/2/31/2; LL/A-31/2;CPS- 1/251/2.

Metric characters: TL (mm)- 153.0; SL (mm)-120.0; HL (mm)-27.0.\% SL: HL-22.5; HD-18.3; HW15.0; BDD-33.3; BDA-20.8; BWD-18.3; BWA-11.7; PRD-45.8; POD-53.3; PRP-24.2; PRPL-48.3; PRA-71.7; LD-22.5; LP-18.3; LPL-17.5; LA-13.3; LBD-15.8; LBA-9.2; LBP-5.0; LBPL-5.0; LCP15.8; DCP-12.5; WCP-5.8; DAV-5.0; DVV-21.7; DP-PL-25.8; DPL-A-25.0. \% HL: HD-81.5; HW66.7; ED-29.6; IOW-48.1; INW-29.6; WGM-33.3; STL-33.3.

Other features: Last unbranched ray of dorsal fin non- osseous, weak and smooth; body with silvery flanks and back light dark; during breeding season male develops long filamentous process on dorsal fin.

\subsection{Gonoproktopterus kurali (Menon \& Rema Devi)}

Hypselobarbus kurali Menon \&Remadevi, 1995, Journal of Bombay natural History society, 92 (3): 389- 393 (Type locality: Mallappally, Manimala River, Kerala).

Diagnosis: Last unbranched dorsal ray weak and smooth; Lateral line with 42 scales; two pairs of maxillary barbels present, the upper pair seemingly absent as it is hidden under labial groove. Caudal fin is with an oblique red band bordered with black; a black band present outer to operculum.

Meristic counts: D-I, 9 ; P-I, 13-14; V-I, 8 ; A-I, 6 ; C-19; LLS-42 ; PDS-13 ; PRPLS-10; PRAS-21 ; LL/D-81/2; LL/V- 41/2; L/ Tr- 81/2/61/2; LL/A-51/2; CPS-7.

Metric characters: TL (mm)- 236.5; SL (mm)-184.0; HL (mm) - 48.5. \% SL: HL- 26.4; HD-15.8; HW-15.5; BDD-21.7; BDA-15.8; BWD-18.0; BWA-12.2; PRD-47.3; POD-49.5; PRP- 24.0; PRPL47.8; PRA-72.3; LD-21.7; LP-18.5; LPL-16.3; LA-24.0; LBSOD-14.7; LBA-8.0; LBP-4.3; LBPL4.3; LCP-15.2; DCP-9.2; WCP-5.4; DVV-22.2.\% HL: HD- 59.8; HW-58.8; ED-22.7; IOW- 35.1; INW-24.7; WGM-18.6; STL-45.4

Remarks: Gonoproktopterus kurali was originally described from Mallappally of Manimala River (Menon \&Remadevi, 1995). The present study revealed that G. kurali is abundantly distributed in Mallappally and various regions of Manimala River. Examination of many specimens of this unveiled a distinct sexual dimorphism in this species

\subsection{Garra mullya (Sykes)}

Garra Hamilton- Buchanan, 1822, Fishes of Ganges: 343, 393 (type species, Cyprinus lamta, by subsequent designation).

Diagnosis: Lateral line scales 32; pre dorsal scales 9; a black spot present behind opercular angle; a black band extends from head to tip of tail.

Meristic counts: D- ii, 8; P- i, 12; V- i, 8; A- ii, 5; C- 18; LLS- 33; LL/D- 51/2; LL/V- 31/2; LL/A- 41/2; PRPLS- 8; PRAS- 16; CPS- 7; PDS-10.

Metric features: TL (mm) - 172.0; SL (mm) - 142.0; HL (mm) -30.0.\% SL: HL-21.1; HD-14.8; HW17.0; BDD-20.4; BDA-15.5; BWD-19.7; BWA-11.3; PRD-40.1; POD-49.3; PRP-17.6; PRPL-47.2; PRA- 74.0; LD-18.3; LP-21.8; LPL- 19.7; LA-17.0; LC-22.5; LBD-14.1; LBP- 5.6; LBPL- 5.6; LBA7.0; LBC-12.0; LCP- 14.8; DCP-12.0; WCP-4.2; DP-PL-31.0; DPL- A-24.6; D- AC-21.1; WGM-8.4; LUCL-21.1; LLCL-21.1.\% HL: HD-70.0; HW-80.0; ED-20.0; IOW-43.3; INW-33.3; DOST-90/0; DOD- 100.0 .

Other features: Back blackish green; sides pale green; ventral side yellowish white; pectoral and pelvic fins darker; anal fins hyaline; dorsal and caudal fins pale dark.

\subsection{Mesonoemacheilus triangularis (Day)}

Nemacheilus triangularis Day, 1865, Proc. Zool. Soc. London: 295 (type locality; Mundakkayam, Kerala)

Diagnosis: 14 oblique, black bordered lateral bands present on sides; a black elliptical patch is present at the caudal fin base; dorsal fin with less than 10 branched rays.

Meristic counts: D- ii, 7; A- ii, 5; V- ii, 5; P- I, 8; C- 21. 
Metric characters: TL (mm) -74.0; SL (mm)- 60.0; HL (mm) -1.2. \% SL: HL-20.0; HD-10.0; BDD20.0; PRD- 48.3; POD-48.3; PRP-53.3; LD-15.0; LP-18.3; LPL-15.0; LBD-16.7; LBA-5.0; LCP11.7; DCP-13.3.\% HL: HD- 50.0; ED- 12.5; STL- 41.7.

Other features: Ground color light greenish in young; it becomes more darker in adults; fins white to yellowish white; dorsal formed of 2-3 rows of black bands and caudal fin with 4 black bands also formed of black dots. A black dot present on the bottom of first dorsal spine. 11 pale black bands present on dorsal side; it is continuous with the 11 adjoining lateral bands; 3 lateral bands have no corresponding bands on dorsal side. 3-5 pale round spots present in between anterior lateral and dorsal bands.

\subsection{Bhavania australis Jerdon}

Platycara australis Jerdon, 1849, Madras J. Lit.Sci..15: 333 (type locality; Walliar, Nilgiris).

Diagnosis: Body depressed anteriorly with flattened ventral profile and laterally compressed posterior half; eyes and nostrils located on the dorsal side of head; two antrose papillae are present below lower jaw; fins fan like; pectoral fin elongated; it reach near to pelvic fin.

Meristic counts: D-i, 7; P- 17; V- 10; A- 5; C- 17.

Metric characters: TL (mm)- 50.5; SL- 40.5; HL (mm)- 8.8. \% SL: HL- 22.2; HD- 11.1; HW-18.5; PRD- 49.4; POD- 53.1; PRP- 22.2; PRPL- 48.1; PRA- 80.2; LP- 28.4; LPL- 22.2; LA- 13.6; LC24.7; LBD- 12.3; LBA- 7.4; LBC- 7.4; DP- PL- 29.6; DPL- A- 35.8; DA- C- 21.0.

\% HL: HD- 51.1; HW- 85.2; ED- 28.4; STL- 51.1; IOW- 39.8; INW- 39.8; WGM- 51.1.

Remarks: Type locality of Bhavania australisis Nilgiris; but the taxonomic details of the present specimen closely resembles with the latter.

\section{DisCuSSION}

Hill streams of Manimala River provide an ideal habitat for many important indigenous fishes. As it provides great heterogeneity in habitat in the form of runs, rapids, falls and pools, fish fauna also exhibits great diversity in their size, shape and color. The river bed of high level regions of Manimala River is mainly rocky; stones and boulders provide suitable habitat of fish for breeding, feeding and hiding places. Barbs, loaches and barils are the main inhabitants of the high level regions of the River. A few rare cat fishes are also found in hill stream stretches.

Destructive fishing practices cause serious threat to ichthyofaunal resources of Manimala River. Use of electric current in the form of electric stickers is being indiscriminately killed many fishes including their fries and fingerlings. Use of pesticides and chemical fertilizers in the nearby agricultural land (which leach into the stream), extract of some toxic plants for fishing purposes are also causing deleterious effect on many hill stream fishes. Removal of instream stones and boulders for building constructions obstructs and destructs the habitat of many fishes. Sincere conservation efforts alone can save the hill stream fishes of Manimala River from destruction.

\section{ACKNOWLEDGEMENT}

The first author acknowledges Principal, Baby John Memorial Government College, Chavara, Kollam, Kerala to carry out this research work.

\section{REFERENCES}

[1] Plamoottil, M, Ichthyodiversity of Manimala River of Kerala, India. Journal of Zoological and Bioscience Research, 2015, 2 (2): 26- 34.

[2] Day, F, The Fishes of Malabar. Bernard Quaritch, London,1865, 185.

[3] Günther, A, Catalogue of fishes in the British Museum. The Annals and Magazine of Natural History, 1864, 3 (14), 374-376.

[4] Plamoottil, M. \& N. P. Abraham, Glyptothorax elankadensis, a new catfish from Kerala, India. Biosystematica, 2012, 6(2): 17- 25.

[5] Plamoottil, M \& N. P. Abraham, Mystus indica and M. heoki, two new catfishes from Kerala, India. Biosystematica, 2013 a, 7 (1): 43-58.

[6] Plamoottil, M. \& N.P. Abraham, Mystus menoni, a new catfish from Kerala, India. International Journal of Pure and Applied Zoology, 2013b, 1 (4): 315-325. 